\title{
CORRECTION
}

\section{Correction to: External confirmation and exploration of the Kigali modification for diagnosing moderate or severe ARDS}

\author{
Veronica Vercesi ${ }^{1,2}$, Luigi Pisani ${ }^{1,3^{*}}$ (D) Patricia S. I. van Tongeren ${ }^{1,4}$, Wim K. Lagrand ${ }^{1}$, Stije J. Leopold ${ }^{3}$, \\ Mischa M. A. Huson ${ }^{5}$, Patricia C. Henwood ${ }^{9}$, Andrew Walden ${ }^{8}$, Marry Smit ${ }^{1}$, Elisabeth D. Riviello ${ }^{6}$, Paolo Pelosi $^{2}$, \\ Arjen M. Dondorp ${ }^{1,3}$ and Marcus J. Schultz ${ }^{1,3,7}$ for the Lung Ultrasound Consortium
}

C 2018 Springer-Verlag GmbH Germany, part of Springer Nature and ESICM

\section{Correction to: Intensive Care Med https://doi.org/10.1007/ s00134-018-5048-5}

In Table 1 of this article, the numerical data were correct but the graphic part was imprecise. The corrected Table 1 is provided here.

Table 1 Diagnostic accuracy of the Kigali modification using the Berlin definition as the reference standard

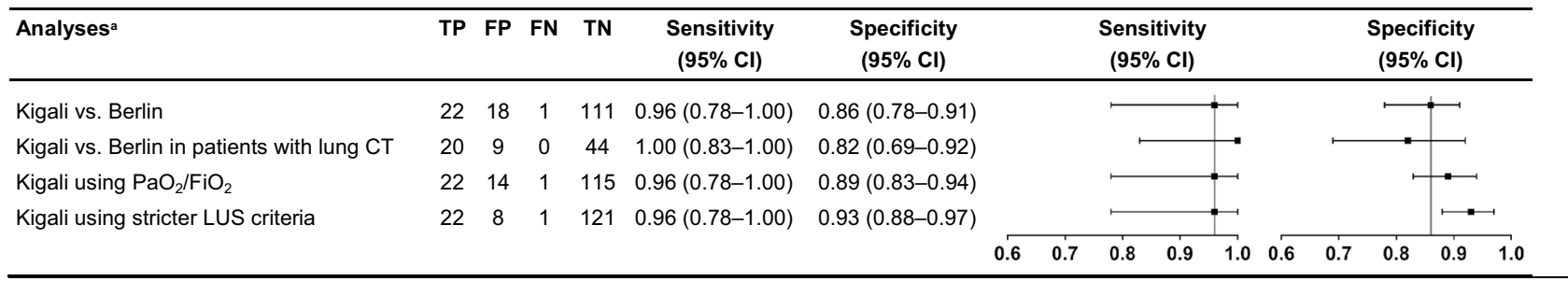

$T P$ true positive, $F P$ false positive, $F N$ false negative, $T N$ true negative, $\mathrm{Cl}$ confidence interval, $C T$ computed tomography, $\mathrm{PaO}_{2}$ oxygen arterial tension, $\mathrm{FiO}{ }_{2}$ fraction of inspired oxygen

a Four analyses were performed: (1) the original Kigali modification vs. the full Berlin definition (152 patients); (2) the original Kigali modification vs. the Berlin

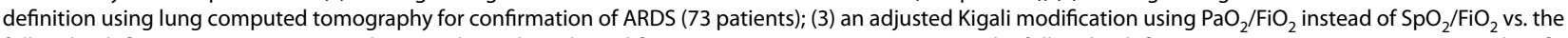
full Berlin definition (152 patients); and (4) an adjusted Kigali modification using stricter LUS criteria vs. the full Berlin definition (152 patients). Patients were classified as having moderate or severe ARDS according to the Kigali modification if they were within 1 week of a known clinical insult (as with the Berlin definition), had a $\mathrm{SpO}_{2} / \mathrm{FiO}_{2}<250$, had on LUS at least two regions with a B (B1 or B2) - or a C pattern on both sides of the chest not explained by effusions, and respiratory failure was not fully explained by cardiac failure or fluid overload. For the fourth analysis using stricter LUS criteria, LUS was "positive" if at least two regions per hemithorax were scored as a B2 or C pattern, or at least three fields as a B1 (i.e., increasing the severity (B2 or C instead of B1, B2 or C) and/or the number of regions ( 3 instead of 2) affected). For more details, see text and the Electronic Supplementary Material

\footnotetext{
*Correspondence: luigipisani@gmail.com

${ }^{1}$ Department of Intensive Care, Academic Medical Center, C3-423,

Meibergdreef 9, 1105 AZ Amsterdam, The Netherlands

Full author information is available at the end of the article 


\section{Author details}

${ }^{1}$ Department of Intensive Care, Academic Medical Center, C3-423, Meibergdreef 9, 1105 AZ Amsterdam, The Netherlands. ${ }^{2}$ Department of Surgical Sciences and Integrated Diagnostics, San Martino Policlinico Hospital, IRCCS for Oncology, University of Genoa, Genoa, Italy. ${ }^{3}$ Mahidol Oxford Tropical Medicine Research Unit (MORU), Mahidol University, Bangkok, Thailand.

${ }^{4}$ Department of Internal Medicine, Tergooi Hospital, Blaricum, Hilversum, The Netherlands. ${ }^{5}$ Department of Internal Medicine, Academic Medical Center,
Amsterdam, The Netherlands. ${ }^{6}$ Division of Pulmonary, Critical Care and Sleep Medicine, Beth Israel Deaconess Medical Center and Harvard Medical School, Boston, MA, USA. ${ }^{7}$ Laboratory of Experimental Intensive Care and Anesthesiology (L.E.I.C.A.), Academic Medical Center, Amsterdam, The Netherlands.

${ }^{8}$ Department of Intensive Care, Royal Berkshire Hospital, Reading, UK.

${ }^{9}$ Department of Emergency Medicine, Brigham and Women's Hospital, Boston, MA, USA.

Published online: 20 February 2018 